\title{
Thermodynamic Discrimination between Energy Sources for Chemical Reactions
}

\author{
Zachary J Schiffer ${ }^{\mathrm{a}}$, Aditya M. Limaye ${ }^{\mathrm{a}}$, Karthish Manthiram ${ }^{\mathrm{a}, *}$ \\ ${ }^{a}$ Department of Chemical Engineering, Massachusetts Institute of Technology, Cambridge, Massachusetts 02139, United \\ States
}

\begin{abstract}
Chemical transformations traverse large energy differences, yet the choice of energy source to drive a chemical reaction is often decided on a case-by-case basis; there is no fundamentally-driven, universal framework with which to analyze and compare the choice of energy source for chemical reactions. In this work, we present a reaction-independent expression for the equilibrium constant as a function of temperature, pressure, and voltage. With a specific set of axes, all reactions can be represented by a single $(x, y)$ point and a quantitative divide between electrochemically and thermochemically driven reactions is visually evident. In addition, we show that our expression has a strong physical basis in work and energy fluxes to the system, although more specific data about reaction operation is necessary to provide a quantitative energy analysis. Overall, this universal equation and facile visualization of chemical reactions enables quick and informed justification for electrochemical versus thermochemical energy sources without knowledge of detailed process parameters.
\end{abstract}

Keywords: Electrochemistry, Chemical thermodynamics, Electrochemical synthesis

In chemical synthesis, the making and breaking of chemical bonds often requires traversing large energy differences. In fact, the basic chemical industry accounts for close to $20 \%$ of total delivered 5 energy consumption in the industrial sector, which itself uses the most delivered energy of any end-use sector globally (54\%) [1, 2]. Traditionally, industrial chemical synthesis has relied on pressure and temperature as driving forces to synthesize chemicals; a reactor requires an exchange of heat and work in order to drive a chemical transformation 3, 2, 4. Yet, with the advent of abundant and accessible renewable electricity, it is attractive to consider driving chemical reactions that are conventionally driven with temperature and pressure with electrical voltage instead $5,6,7,8,9$. Hence, one is confronted with the question, "why should a given chemical reaction be driven preferentially with temperature (thermal energy), pressure (mechanical 20 energy), or voltage (electrical energy)?" The response to this question is generally either broad and qualitative or extremely reaction-specific. Broadly,

${ }^{*}$ To whom correspondence should be addressed. E-mail: karthish@mit.edu

Preprint submitted to ChemRxiv if a reaction is highly endothermic (results in a very positive change in enthalpy), then one may prefer 25 an electrochemical approach that avoids excessively high operating temperatures to shift the equilibrium toward products [10, 9. Additionally, if a reaction requires high pressures to drive conversion to products via Le Chatelier's principle, then one may s prefer using voltage to avoid these excessively high pressures. Technoeconomic analyses are also often used to discriminate between thermochemical and electrochemical driving forces based on feedstock costs and system efficiencies [11, 12, 13, 14, 15, 16. 35 However, these technoeconomic analyses remain focused on specific reactions without investigating the physical basis for the preference of driving force in a general manner. While many of the choices in the driving chemical reactions are determined by to factors such as kinetics, cost, and safety, research and development often begins before estimates of these specific parameters are known. Accordingly, an intermediate-level heuristic for choosing a driving force based on available physical parameters is 45 missing; specifically, one that is simple and intuitive, yet also quantitative and dependable across a wide range of chemical reactions.

September 16, 2020 
We address the question of how to discriminate between energy sources for driving chemical reac50 tions via a theoretical framework built around reaction thermodynamics. Efficiency and thermodynamic limits on extraction of useful work have been studied for centuries (e.g., with the Carnot engine) [17, 18, and more recent work has slowly

55 relaxed ideal constraints to add in real-world practicalities via developments in fields such as endoreversible thermodynamics and finite-time thermodynamics [19, 20]. However, these theories are often built to describe the extraction of energy from so a chemical reaction, e.g., in a combustion engine, and they often still require significant knowledge of specific process parameters such as heat transfer coefficients, compressor efficiencies, thermodynamic paths, etc [21]. In this work, we construct 65 a universal equation to describe and analyze the thermodynamics of chemical reactions driven by temperature, pressure, and voltage. We have focused on these driving forces due to their prevalence in chemical synthesis, although the analysis 70 can be extended to the direct use of photons or mechanochemical methods. We compare heat, mechanical work, and electrical work as energy inputs to a chemical system and find that an ideal, lossless model of energy comparison provides a phys75 ical basis for our non-dimensional thermodynamic parameter analysis. After constructing a universal equation, we then introduce a facile visualization 110 method for comparing chemical reactions, with a focus on redox reactions (voltage is generally not an option for non-redox reactions), and show a clear divide between chemical reactions traditionally driven by elevated temperatures and pressures 115 in industry and reactions that rely on electrical voltage. Our approach provides a simple, universal 85 method to justify using temperature, pressure, or voltage as a driving force for a chemical reaction, and our analysis can be leveraged by researchers in 120 a broad range of fields to help determine the important systems-level choice of thermodynamic driving 90 force.

\section{Results and discussion}

\section{Non-dimensionalization of reaction equilibrium}

We are interested in comparing the effects of temperature, pressure, and voltage as driving forces for 130 95 shifting the equilibrium of a chemical reaction. Accordingly, we start with a chemical reaction that has some defined stoichiometry given by,

$$
\sum_{i} \nu_{i} A_{i}=0
$$

where $\nu_{i}$ are the stoichiometric coefficients for chemical species $A_{i}$. Chemical equilibrium at constant temperature $(T)$, pressure $(P)$, and voltage $(E)$ provides the constraint,

$$
\sum_{i} \nu_{i} \mu_{i}(T, P, E)=0
$$

where $\mu_{i}$ are the species chemical potentials. Assuming that the system is an ideal mixture of gases and that $\Delta C_{P, \text { rxn }} \equiv \sum_{i} \nu_{i} C_{P, i}=0$, the equilibrium constant, $K$, is a simple function of thermodynamic variables (Supplemental Derivation S1) [22, 23, 24,

$$
\begin{aligned}
\log _{e} K= & \frac{-\Delta G_{\mathrm{rxn}}(T, P, E)}{R T} \\
= & -\frac{\Delta H_{\mathrm{rxn}}^{0}}{R T}-\frac{n_{e^{-}} F E}{R T}-\Delta n_{\mathrm{rxn}} \log _{e} \frac{P}{P^{0}} \\
& +\frac{\Delta S_{\mathrm{rxn}}^{0}}{R},
\end{aligned}
$$

where $\Delta H_{\mathrm{rxn}}^{0}$ and $\Delta S_{\mathrm{rxn}}^{0}$ are the enthalpy and entropy of reaction, respectively, at ambient conditions (namely no applied voltage, $T=T^{0}=$ $298.15 \mathrm{~K}$, and $P=P^{0}=1$ bar), $R$ is the ideal gas constant, $n_{e^{-}}$is the minimum number of electrons necessarily transferred in the overall reaction (Supplemental Derivation S1), and $\Delta n_{\mathrm{rxn}} \equiv \sum_{i \in \text { gas }} \nu_{i}$. Although we assume for simplicity that all of our components are gases (with a few exceptions for pure liquids and solids), the extension to liquids, dissolved species, and solids is not difficult to incorporate when going through the full derivation (Supplemental Derivation S1). Equation 3 is a familiar description of the equilibrium constant with one major difference: we have defined $K \equiv \prod_{i \in \text { gas }} y_{i}^{\nu_{i}}$, with $y_{i}$ being the mole fraction of each component in the gas phase, instead of the more traditional $K=\prod_{i} p_{i}^{\nu_{i}}$, where $p_{i}$ is the partial pressure of 25 a species (i.e., the activity of an ideal gas) [25]. In this work, the equilibrium constant is defined by equation 3 instead of the traditional definition so that pressure will be explicitly included in the expression. Through equation 3 and proper stoichiometry normalization, we can better compare reaction equilibriums based on a more consistent relationship between mole fractions and $K$ that is 
not present when $K$ is written in terms of activity (e.g., partial pressures) instead of mole fractions.

The practically relevant quantity for describing a chemical reaction is conversion, not the equilibrium constant. However, conversion will be dependent on the exact reaction equation and not a universal function. To keep the analysis general, the equilibrium constant, $K$, will be used as a proxy for conversion since conversion is a strictly increasing, sigmoidal function of $K$ ranging from 0 to 1 (Supplemental Derivation S2).

Unfortunately, the expression for the equilibrium constant given by equation 3 does not allow for facile comparison of temperature, pressure, and voltage in its current form since the quantities $\Delta H_{\mathrm{rxn}}^{0}, \Delta S_{\mathrm{rxn}}^{0}, \Delta n_{\mathrm{rxn}}$, and $n_{e^{-}}$are reactionspecific, preventing a general analysis of chemical reaction equilibrium. To facilitate a comparison, the equilibrium constant expression can be non-dimensionalized to remove any specifics about the chemical reaction. Non-dimensionalization is a useful tool for solving differential equations in imits and for quickly determining characteristic properties of a system such as time and length; for these reasons it finds wide use across the fields of chemical engineering, physics, fluid dynamics, etc [26]. In the case of a chemical reac, non-dimensionalization combines the reactionspecific details of the system with the reaction operating conditions to create new variables that are reaction-independent and scale simply with the thermodynamic driving forces of interest (Figure Supplemental Derivation S3). Traditional nondimensionalization, e.g., of reaction-diffusion systems, relies on the structure of a differential equation to provide the non-dimensional groupings; an algebraic equation such as equation 3 does not groupings, and non-dimensionalization must instead rely on underlying physical intuition.

Equation 3 can be non-dimensionalized to achieve a universal expression for how thermal, mechanical, and electrical energy shift the chemical equilibrium through non-dimensional temperature 195 $(T \rightarrow \Theta)$, pressure $(P \rightarrow \Pi)$, and voltage $(E \rightarrow \Psi)$,
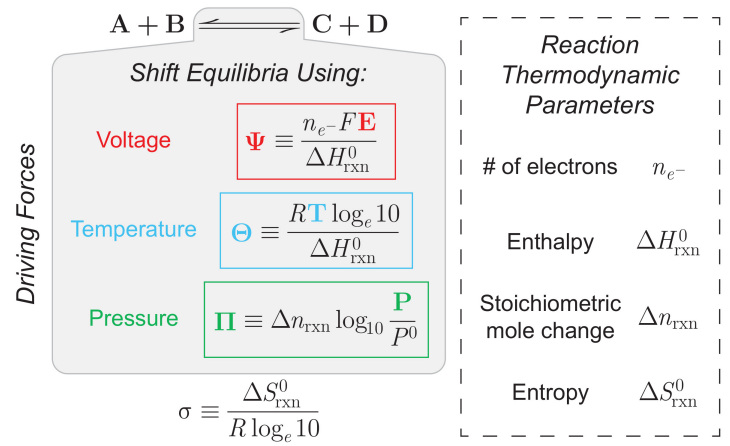

Figure 1: Non-dimensionalization scheme for thermodynamic variables. Given a generic reaction, here shown as the conversion of reactants $\mathrm{A}$ and $\mathrm{B}$ to products $\mathrm{C}$ and $\mathrm{D}$, reaction-specific thermodynamic parameters (right) and the reaction operation conditions (left) can be combined to obtain non-dimensional groupings that scale with the thermodynamic driving forces and enable non-dimensionalization of equation 3 to obtain equation 4

$$
\begin{aligned}
\Theta & \equiv \frac{R T \log _{e} 10}{\Delta H_{\mathrm{rxn}}^{0}}, \\
\Pi & \equiv \Delta n_{\mathrm{rxn}} \log _{10} \frac{P}{P^{0}}, \\
\Psi & \equiv \frac{n_{e^{-}} F E}{\Delta H_{\mathrm{rxn}}^{0}}, \\
\sigma & \equiv \frac{\Delta S_{\mathrm{rxn}}}{R \log _{e} 10}, \\
\log _{10} K & =-\frac{1}{\Theta}-\frac{\Psi}{\Theta}-\Pi+\sigma .
\end{aligned}
$$

Note that we have chosen to use $\Theta \propto T$ instead of a potentially "natural" quantity $\Theta \propto 1 / T$ so that changes in $\Theta$ are more intuitively interpretable [27] . One of the advantages of equation 4 is that all reactions collapse onto simple plots that show the equilibrium constant, a proxy for reaction conversion, as a function of non-dimensional thermodynamic driving forces (Figure 2). This visualization reveals that crossing equilibrium contours with pressure requires a larger relative increase in thermodynamic driving force than crossing equilibrium contours with temperature or voltage (further supporting analysis of contours and derivatives provided in Supplemental Derivation S4). This discrepancy is magnified by the fact that the scaling of $\Pi$ is logarithmic with pressure whereas the scalings of $\Theta$ and $\Psi$ are linear with temperature and voltage, respectively.

So far, the analysis has relied on the mathematical form of our non-dimensional parame- 

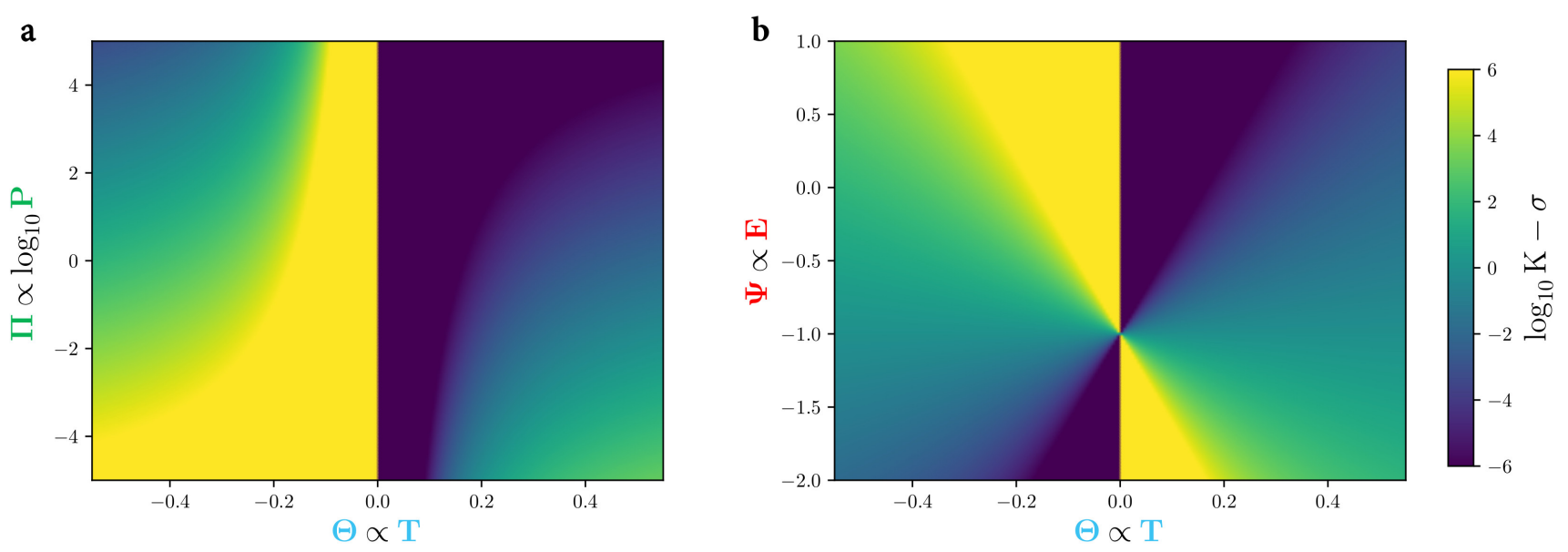

Figure 2: Plots of equation 4 Every chemical reaction can be mapped onto these universal plots. When no voltage is applied $(\Psi=0)$, contours showing how pressure and temperature affect reaction equilibrium are visualized (a). At ambient pressure $(\Pi=0)$, contours showing how voltage and temperature affect the reaction equilibrium are visualized (b). Contours of how voltage and pressure affect the equilibrium at $298.15 \mathrm{~K}$ (ambient temperature) are not as simple to visualize; there is a $\Theta^{\text {ambient }}$ term in these contours that make the plot less useful because it is reaction dependent and not generalizable (these plots and full discussion in Supplemental Derivation S4). Crossing the constant $K$ contours using pressure requires a larger relative change in non-dimensional driving force than it does using voltage or temperature. Note that the colorbar axis is $\log _{10} K-\sigma$ so that the plot remains independent of reaction; different reactions will essentially provide a constant shift from $\sigma$ that does not change the shape of the plot. Additionally, that crossing $\Theta=0$ for a given reaction is impossible since the sign of $\Theta$ is determined by the reaction enthalpy (a fixed quantity assuming $\Delta C_{P, \mathrm{rxn}}=0$ ).

ters. In practice, there are many alternative nondimensional groupings with additional constant factors or functional forms that would change this 200 analysis. However, these non-dimensional thermodynamic parameters (Figure 1) are not only 225 convenient from a mathematical perspective, but also represent physical groupings related to analogous work and energy fluxes, discussed below, such that conclusions drawn from analysis of the nondimensional thermodynamic parameters are physically relevant.

\section{Work and energy exchange}

A direct comparison between temperature, pressure, and voltage is difficult since each driving force has different units. Even with our non-dimensional scalings, there is no reason to assume a priori that these non-dimensional parameters have any physical meaning and can be directly compared ${ }_{235}$ o each other. Instead, a metric for comparing driving forces on equal footing would be to compare work and energy exchanges of the system; this comparison will provide a physical basis for our non-dimensional parameters so that we can directly 240 compare them.
In practice, no general method to convert between thermodynamic parameters and work exists since heat and work are path functions. However, if the system does not have any energy losses, the overall, steady-state energy exchanges of the system must obey the law of energy conservation,

$$
W_{\mathrm{M}}+W_{\mathrm{E}}+Q=\Delta H_{\mathrm{rxn}}^{0} \cdot z,
$$

where $z$ is the reaction conversion at equilibrium, a function of $K$ and defined between 0 and 1. A more convenient form of equation 5 results from - cimensionalization of the work terms with the reaction enthalpy as the characteristic energy of the system (Supplemental Derivation S5),

$$
\Omega_{W_{\mathrm{M}}}+\Omega_{W_{\mathrm{E}}}+\Omega_{Q}=z .
$$

Ignoring the exact functional form of the work terms for now and assuming that the system is driven by either pressure or voltage individually, but not both simultaneously, the constraint imposed by equation 6 has the geometric form of a plane in $\Omega_{W_{i}}-\Omega_{Q}$ space (Figure 3). Accordingly, for a system with no energy losses, any energy input will result in an equivalent change in conversion, regardless of the energy source. If additional 


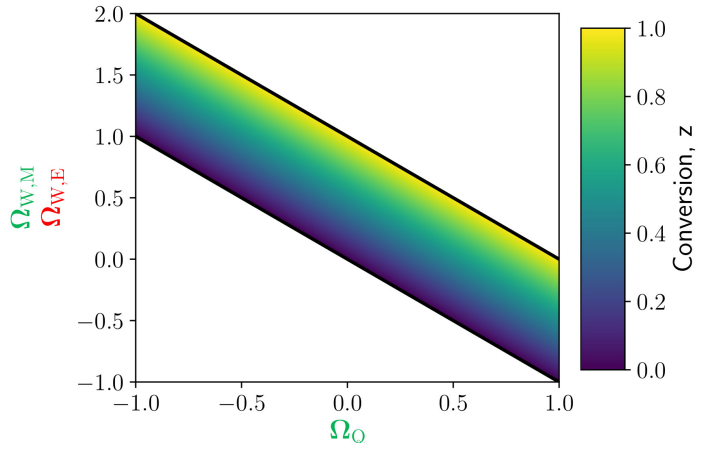

Figure 3: Conversion, $z$, as a function of various energy inputs assuming no energy losses in the system. In this system, either pressure or voltage is utilized, but not both simultane- 275 ously. In the absence of losses, all energy exchanges produce the same change in conversion, regardless of the source of the energy. Thus, in the absence of additional, reactionand process-specific information, this energy analysis simply provides a physical basis for the non-dimensional analysis, as discussed in the main text.

information is available, such as the cost of electricity, efficiency of heat flux, compressor losses, etc., there are a multitude of thermodynamic and technoeconomic heuristics that can lead to a quantitative conclusion, but these are beyond the scope of this work.

In the absence of more information, there are still important insights to glean from the functional s of the work and heat inputs. In addition to the previous assumptions (ideal gas mixture and $\left.\Delta C_{P, \text { rxn }}=0\right)$, additional assumptions are necessary to convert from thermodynamic parameters to work and energy fluxes: (1) the reactor is isothermal, isobaric, and does not exchange mechanical work with the environment, and (2) the processes that bring the inputs to the operating conditions and bring the outputs back to ambient conditions have access to a single heat bath at some $T_{\text {bath }}$. Given these assumptions, as well as assuming unit efficiency of every process, the total energy and work exchanges with the overall system are functions of the previous thermodynamic parameters, the conversion, $z=z(K)$, which is a function of the equilibrium constant, and the single non-dimensional thermal bath temperature, $\Theta_{\text {bath }} \equiv \frac{R T_{\text {bath }} \log _{e} 10}{\Delta H_{\mathrm{rxn}}^{0}}$ (Supplemental Derivation S5) [28, 29, 30, 31,

$$
\begin{aligned}
& \Omega_{W_{\mathrm{E}}}=-z(K) \Psi \\
& \Omega_{W_{\mathrm{M}}}=-z(K) \Theta_{\text {bath }} \Pi \\
& \Omega_{Q}=z(K)\left(\Psi+\Theta_{\text {bath }} \Pi+1\right)
\end{aligned}
$$

Without additional practical information, our energy analysis is qualitative, and these equations by

270 ical work, electrical work, or heat. However, these equations do provide justification for our previous analysis. We initially began our reaction analysis by non-dimensionalizing temperature, pressure, and voltage to remove any reaction-specific quantities from the equilibrium expression given in equation 3. The non-dimensionalization was intuitive yet somewhat arbitrary, and there was no indication that the non-dimensional parameters $(\Theta, \Pi$, and $\Psi$ ) had any physical meaning. The energy analysis presented here, however, reveals that the non-dimensional electrical work $\left(\Omega_{W_{\mathrm{E}}}\right)$ will scale directly with non-dimensional voltage $(\Psi)$, the nondimensional mechanical work $\left(\Omega_{W_{\mathrm{M}}}\right)$ will scale directly with non-dimensional pressure $(\Pi)$, and the heat flux is a convolution of all the energy inputs but has a clear characteristic energy given by $\Delta H_{\mathrm{rxn}}^{0}$, which was used to non-dimensionalize temperature $(\Theta)$. Accordingly, although the previous analysis dealt with non-dimensional driving forces that were not, a priori, comparable, this energy analysis reveals that these non-dimensional quantities are reasonable proxies for work and energy exchanges and that our non-dimensional analysis has a strong physical basis. Direct comparisons of the non-dimensional thermodynamic parameters therefore correspond to comparisons of analogous energy and work exchanges, validating conclusions drawn from such direct comparisons.

\section{Example chemical reactions on universal plot}

While the universal colormaps of equation 4 are interesting on their own (Figure 2), contour lines of constant $K$ for a specific reaction help visualize the thermodynamics of that reaction. This is the advantage of plotting the equilibrium constant in non-dimensional space: instead of qualitatively looking at endo- vs. exo-thermicity or analyzing a specific reaction's equilibrium constant at various temperatures, pressures and voltages, we can 
forces on a single set of axes since the thermodynamic landscapes for individual reactions all collapse onto a single plot in non-dimensional space (Figure 2). Two well-studied reactions are ammonia synthesis (Reaction R 1 industrially known as 360 the Haber-Bosch process),

$$
\frac{1}{2} \mathrm{~N}_{2}+\frac{3}{2} \mathrm{H}_{2} \longrightarrow \mathrm{NH}_{3}
$$

and water splitting (Reaction $\mathrm{R} 2$,

$$
\mathrm{H}_{2} \mathrm{O} \longrightarrow \mathrm{H}_{2}+\frac{1}{2} \mathrm{O}_{2} \text {. }
$$

The constant $K$ contours for these reactions can be plotted on the universal colormaps and com- 370 pared (Figure 4) 32. Using the reaction thermodynamic properties, dimensional parameters $(T, P$, and $E$ ) are shown for each reaction on the secondary axes. In addition to helping visualize the equilibrium constants for these reactions in stan- 375 dard units, these secondary axes demonstrate that the non-dimensional axes span a sufficient range of operating conditions for most reactions. The key points to consider with these plots are: (1) the red dot represents ambient conditions, and the 380 horizontal distance to $\Theta=0$ is inversely proportional to the enthalpy of reaction; (2) the red vertical line attached to each ambient conditions point on the pressure-temperature plots (Figure 4 and c) represents an increase of an order of magnitude 385 in pressure; (3) the vertical distance from the red dot to the solid pink line $(K=1)$ on the voltagetemperature plots (Figure $4 \mathrm{~b}$ and $\mathrm{d}$ ) is the nondimensional equilibrium potential of the reaction.

For the case of ammonia synthesis (Figure $4 \mathrm{a}$ and 390 b), the thermodynamic equilibrium favors full conambient conditions; however, kinetics mandate the use of an elevated operating temperature and pressure. This reaction is an important example of the utility of thermodynamic analyses even for reac345 tions where kinetics dictate operating conditions. The scaling of the axes (seen by the secondary axes) demonstrates that crossing equilibrium contours and moving around the thermodynamic equilibrium space with temperature and pressure are easible at practical operating conditions for am- 400 monia synthesis. In other words, at a temperature at which the kinetics are favorable for ammonia synthesis, the visualization demonstrates that pressure can allow us to easily move through thermo- fact that ammonia synthesis is commercially practiced at elevated pressures that enable higher equilibrium conversions. This is in contrast with water splitting (Figure 4te and d), for which the visualization clearly demonstrates that increasing pressure decreases conversion, and enormous temperatures are necessary to achieve meaningful equilibrium conversions. However, voltage remains a powerful tool, since approximately $1.2 \mathrm{~V}$ is sufficient to 65 drive water splitting, a feasible amount compared to the high temperature or low pressure necessary otherwise. This analysis is currently limited to thermodynamic equilibrium considerations, while in reality temperature, pressure, and voltage also play a role in kinetics, cost, selectivity, and safety, all of which strongly influence the trade-offs between thermochemistry and electrochemistry. These other factors are beyond the scope of this analysis as we aim to describe broad trends in driving forces for chemical reactions using a thermodynamic framework. Even without knowledge of these other factors, a thermodynamic analysis reveals whether high conversion is even physically possible, a prerequisite to engineering reactivity given operating conditions dictated by kinetics and other non-thermodynamic constraints. This continues to motivate the search for catalysts that are active at lower temperatures in the case of ammonia synthesis, while telling us not to search for thermochemical water splitting catalysts at ambient conditions due to thermodynamic restrictions.

While these universal colormaps are useful for visualizing individual reactions, they are not ideal for comparing multiple reactions because they require unique constant $K$ contours for each reaction, meaning that multiple reactions would quickly obscure each other. Instead, the axes can be redefined such that every reaction has the same $K$ contours, facilitating direct comparison between different reactions.

Visual comparison and analysis of chemical reactions

Instead of using the non-dimensional groups derived above as axes, in which each reaction has a distinct set of $K$-contours, a simple variable transformation can collapse these to a single set of equilibrium $K$ contours for all reactions (Figure 5). Specifically, the $x$-axis is transformed to $1 / \Theta$ and the $y$-axis is transformed to $\Psi / \Theta+\Pi-\sigma$ (Supplemental Derivation S6). In general, we assume that 

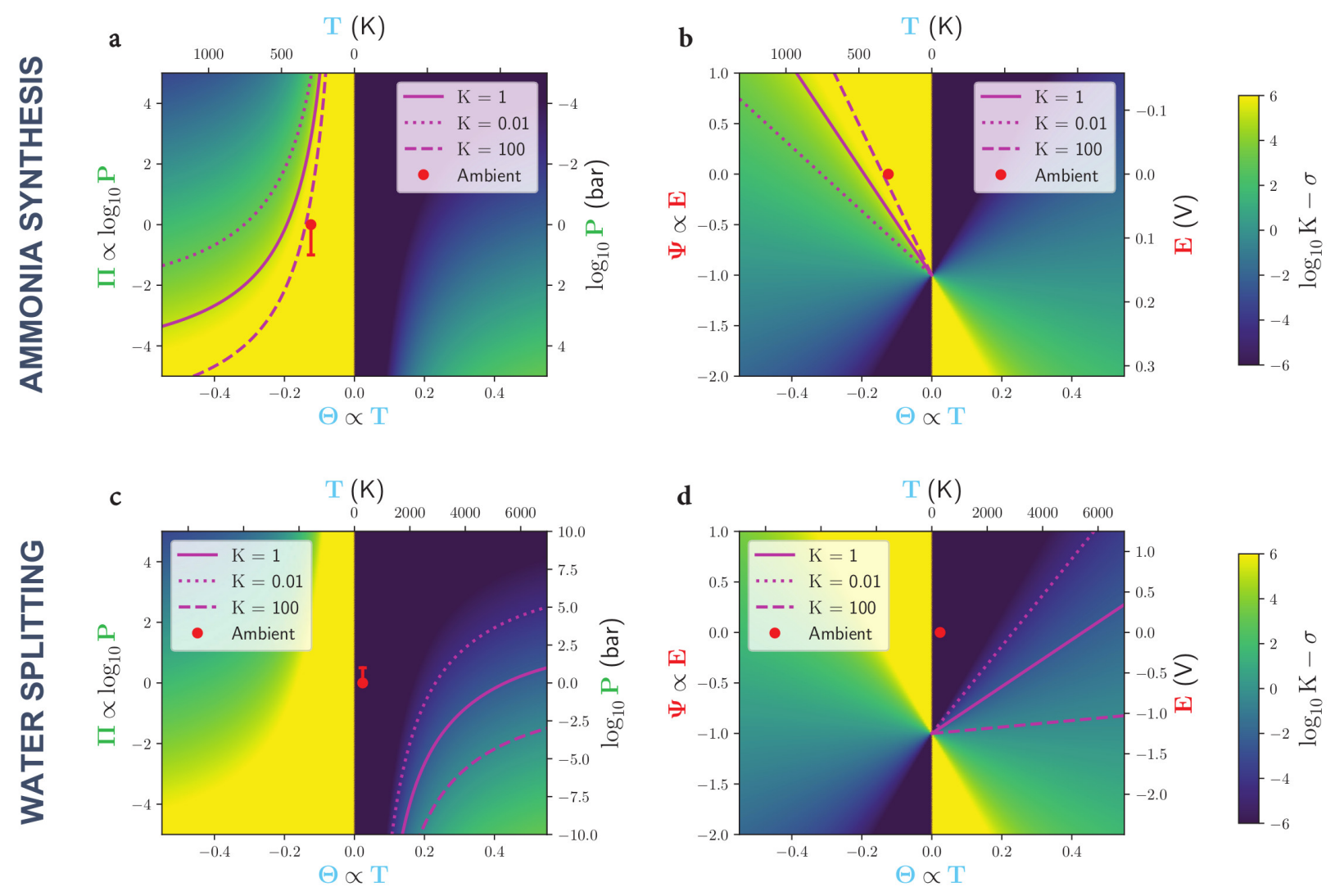

Figure 4: Plots of equation 4 for ammonia synthesis (Reaction $\mathrm{R} 11$ and water splitting (Reaction $\mathrm{R} 2$ 2), using the given stoichiometry. Pink lines indicate constant $K$ contours for each reaction, the red dots represent ambient conditions, and the vertical line extending from each red point in the thermochemistry plots (a and $\mathbf{c})$ represents an increase in pressure of one order of magnitude. In particular, the horizontal distance from the red dot to $\Theta=0$ is inversely proportional to the enthalpy of reaction, and the vertical distance between the red dot and the solid pink line on the electrochemistry plots (b and $\mathbf{d})$ represents the non-dimensional equilibrium potential. Dimensional thermodynamic parameters on the secondary axes demonstrate that the axes span a sufficient range of operating conditions and are reaction dependent. Dimensional parameters also show how reactions cannot cross $\Theta=0$ since that would correspond to a switch in sign of $\Delta H_{\mathrm{rxn}}^{0}$, a quantity fixed by the reaction (assuming $\Delta C_{P, \text { rxn }}=0$ ). Temperature and pressure enable facile movement in thermodynamic space for ammonia synthesis, whereas voltage is necessary to drive water splitting.

in addition to temperature, either voltage or pressure is being used to drive the reaction, not both simultaneously, which results in either $\Pi=0$ or $\Psi=0$, respectively.

As depicted, in these axes a change in pressure or voltage corresponds to a vertical movement relative to the reaction point and an increase (decrease) in $T_{420}$ corresponds to a movement toward (away from) the point $(0, \Pi-\sigma)$ (Figure 5a, Supplemental Derivation S6). These new, composite axes allow for the direct comparison of chemical reactions since all reactions have the same $K$ contours, with each reac- ${ }_{425}$ tion at ambient conditions represented by a single point,

$$
\begin{aligned}
(x, y)_{\mathrm{rxn}}^{\mathrm{ambient}} & =\left(\frac{1}{\Theta^{\text {ambient }}},-\sigma\right) \\
& =\left(\frac{\Delta H_{\mathrm{rxn}}^{0}}{R T^{0} \log _{e} 10},-\frac{\Delta S_{\mathrm{rxn}}^{0}}{R \log _{e} 10}\right),
\end{aligned}
$$

where $T^{0}=298.15 \mathrm{~K}$. For each reaction point at ambient conditions, the distance from the reaction point to $x=0$ is proportional to $\Delta H_{\mathrm{rxn}}^{0}$, the distance to $y=0$ is proportional to $\Delta S_{\mathrm{rxn}}^{0}$, and the distance to the solid black line $(K=1$, given by $y=-x)$ is proportional to the dimensional equilibrium potential of the reaction (Figure 5b, Supplemental Derivation S7). 

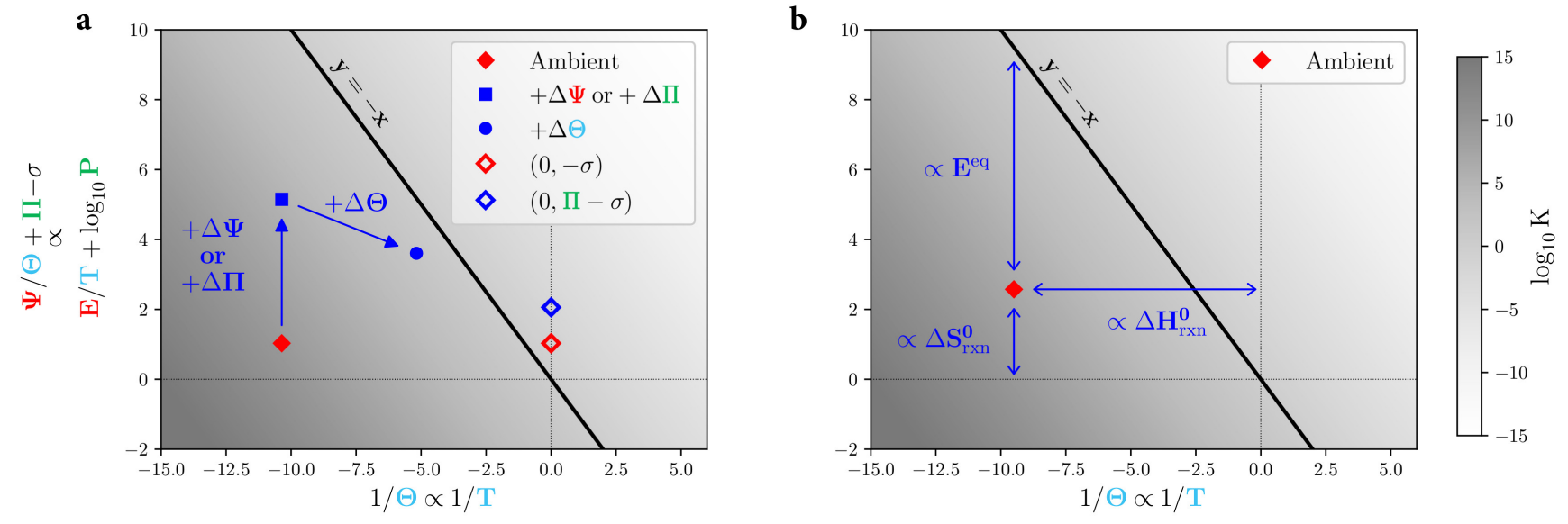

Figure 5: Redefined axes such that all reactions have the same equilibrium $K$ contours. A contour corresponding to $K=1$ is indicated with a diagonal black line $(y=-x)$. The filled red diamonds represent example reaction points at ambient conditions given by equation 8 An increase in either pressure $(\Pi)$ or voltage $(\Psi)$ is a vertical movement on these axes (a, new reaction point given by blue square) and an increase in temperature $(\Theta)$ is a movement towards the point $(0, \Pi-\sigma)(\mathbf{a}$, new reaction point given by blue circle, movement along the line connecting to the empty blue diamond). As shown, the $x$-coordinate of each point is proportional to $\Delta H_{\mathrm{rxn}}^{0}$, the $y$-coordinate of each point is proportional to $\Delta S_{\mathrm{rxn}}^{0}$, and the vertical distance from each point to the solid black line $(K=1$, given by $y=-x)$ is proportional to the dimensional equilibrium potential of the reaction (b). Further details in Supplemental Derivations S6 and S7.

When multiple reactions are plotted on these axes, a clear divide appears between those that are conventionally driven thermochemically versus electrochemically (Figure 6). Reactions to the left of 460 the $K=1$ line $(y=-x)$ are already thermodynamically favorable at ambient conditions. Any adjustment to the reaction conditions (e.g., an increase in temperature to improve kinetics) must keep the reaction as far left as possible to maintain thermo- 46 dynamic favorability. If the reaction point is near or to the right of the $K=1$ line $(y=-x)$, then pressure or temperature can practically cross the equilibrium contours only if the reaction point is within a reasonable distance to the $K=1$ line. In particular, to use pressure to drive conversion, the vertical distance from the reaction point to the line $y=-x$ must be within a couple of orders of 445 magnitude of pressure (a version of Figure 6 with pressure effects depicted for each reaction is shown in Supplemental Derivation S6).

If the reaction point lies to the right of the $K=1$ line $(y=-x)$, then the reaction can be driven to quantifiable conversion using just temperature when the horizontal distance from the 480 reaction point to $y=-x$ is sufficiently small and the reaction point does not lie in the topright quadrant; in that quadrant, the $\Delta H_{\mathrm{rxn}}^{0}$ and $\Delta S_{\mathrm{rxn}}^{0}$ conspire to make the $K=1$ contour unreachable with temperature. The horizontal dis- 485 tance to $y=-x$ is quantified by the temperature, $T^{\mathrm{eq}}$, when $K\left(P=1 \mathrm{bar}, T=T^{\mathrm{eq}}\right)=1$, namely when $T^{\mathrm{eq}}=\Delta H_{\mathrm{rxn}}^{0} / \Delta S_{\mathrm{rxn}}^{0}$. Due to physical practicalities, the operating temperature must be within a factor of ca. 5 of the ambient temperature $(\sim 1500 \mathrm{~K})$. Mathematically, this translates to $T^{\text {eq }} / T^{\text {ambient }}=1 /\left(\sigma \Theta^{\text {ambient }}\right) \leq 5$. For a reaction point given by $(x, y)_{\mathrm{r} \times n}^{\mathrm{ambient}}$ on these axes, the reaction can practically be driven by temperature alone when $1 /\left(\sigma \Theta^{\text {ambient }}\right)=-x / y \leq 5$, approximately (green shading on Figure 6).

Voltage, however, is particularly well suited to drive chemical reactions that are far from the $K=1$ line since even large values of $\Psi / \Theta$ generally correspond to voltages of order $1 \mathrm{~V}$ (Supplemental Derivation S4). Thus, this visualization quantitatively supports our intuition, namely that reactions with large, positive values of $\Delta H_{\mathrm{rxn}}^{0}$ (highly endothermic) are generally better driven by voltage. In particular, reactions which require large excursions on these non-dimensional axes can generally only be done electrochemically (Figure 6, blue points). Those requiring small excursions on these axes can generally be done either electrochemically or thermochemically, and these reactions often are driven with temperature and pressure due to industrial expertise and convenience (Figure 6, pink points). For reactions that could be driven either thermochemically or electrochemically, reaction- 
$1 / 2 \mathrm{~N}_{2}+3 / 2 \mathrm{H}_{2} \rightarrow \mathrm{NH}_{3}$

> $\mathrm{NH}_{3} \rightarrow 1 / 2 \mathrm{~N}_{2}+3 / 2 \mathrm{H}_{2}$

$\Delta \mathrm{H}_{2}+\mathrm{CO}+\mathrm{C}_{2} \mathrm{H}_{4} \rightarrow \mathrm{C}_{3} \mathrm{H}_{6} \mathrm{O}$

* $\mathrm{C}_{3} \mathrm{H}_{8} \rightarrow \mathrm{C}_{3} \mathrm{H}_{6}+\mathrm{H}_{2}$

$<2 \mathrm{H}_{2} \mathrm{~S}+\mathrm{O}_{2} \rightarrow 2 \mathrm{~S}(\ell)+2 \mathrm{H}_{2} \mathrm{O}$

$6 \mathrm{CH}_{4} \rightarrow \mathrm{C}_{6} \mathrm{H}_{6}+9 \mathrm{H}_{2}$

- $\mathrm{CO}_{2}+3 \mathrm{H}_{2} \rightarrow \mathrm{CH}_{3} \mathrm{OH}+\mathrm{H}_{2} \mathrm{O}$

- $\mathrm{CO}+2 \mathrm{H}_{2} \rightarrow \mathrm{CH}_{3} \mathrm{OH}$

$\mathrm{CO}_{2}+\mathrm{CH}_{4} \rightarrow 2 \mathrm{H}_{2}+2 \mathrm{CO}$

- $\mathrm{H}_{2} \mathrm{O} \rightarrow \mathrm{H}_{2}+1 / 2 \mathrm{O}_{2}$

$\mathrm{CO}_{2} \rightarrow \mathrm{CO}+1 / 2 \mathrm{O}_{2}$

- $\mathrm{N}_{2}+3 \mathrm{H}_{2} \mathrm{O} \rightarrow 2 \mathrm{NH}_{3}+3 / 2 \mathrm{O}_{2}$

$2 \mathrm{CO}_{2}+2 \mathrm{H}_{2} \mathrm{O} \rightarrow \mathrm{C}_{2} \mathrm{H}_{4}+3 \mathrm{O}_{2}$

$\rightarrow \mathrm{C}_{2} \mathrm{H}_{4}+\mathrm{H}_{2} \mathrm{O} \rightarrow \mathrm{C}_{2} \mathrm{H}_{4} \mathrm{O}+\mathrm{H}_{2}$

$\Delta 2 \mathrm{NaCl}(\mathrm{s})+2 \mathrm{H}_{2} \mathrm{O}(\ell) \rightarrow \mathrm{H}_{2}+\mathrm{Cl}_{2}+2 \mathrm{NaOH}(\mathrm{s})$

\ $2 \mathrm{Al}_{2} \mathrm{O}_{3}(\mathrm{~s})+3 \mathrm{C}(\mathrm{s}) \rightarrow 4 \mathrm{Al}(\mathrm{s})+3 \mathrm{CO}_{2}$

$+2 \mathrm{C}_{3} \mathrm{H}_{3} \mathrm{~N}+\mathrm{H}_{2} \mathrm{O} \rightarrow \mathrm{C}_{6} \mathrm{H}_{8} \mathrm{~N}_{2}+1 / 2 \mathrm{O}_{2}$

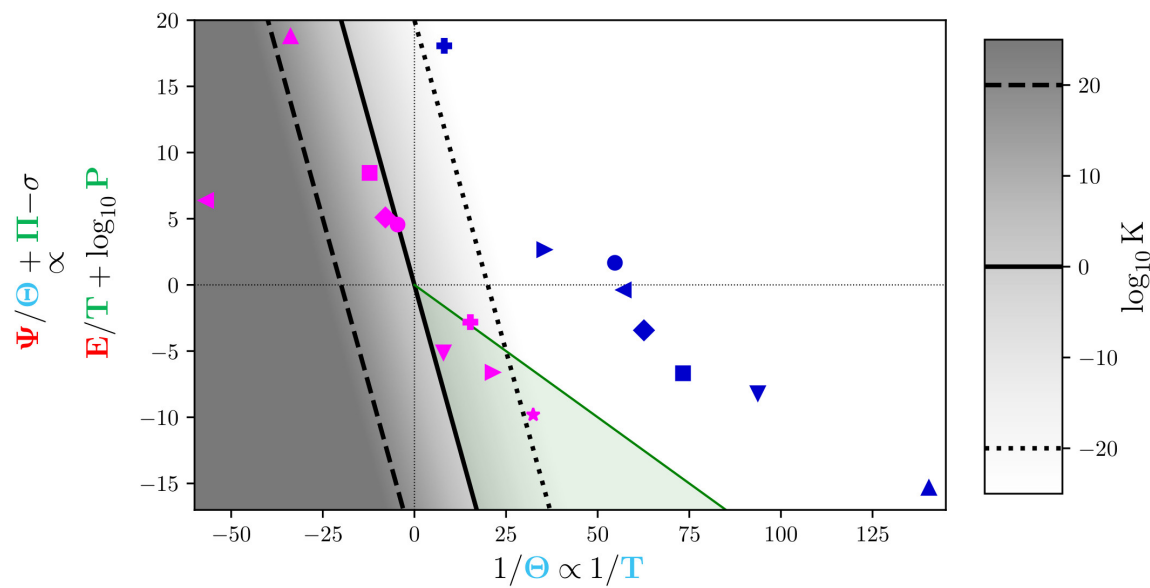

Figure 6: Comparison of multiple reactions on the same axes. Each point represents a chemical reaction as per equation 8 with color denoting whether the reaction is typically conducted thermochemically (pink) or electrochemically (blue). Each reaction point is such that $(x, y)^{\text {ambient }} \propto\left(\Delta H_{\mathrm{rxn}}^{0},-\Delta S_{\mathrm{rxn}}^{0}\right)$ and can be interpreted as described in Figure 5 (addition of vertical lines representing the effect of pressure analogous to that in Figure 4 shown in Supplemental Derivation S6). The green shading indicates the area where an elevated temperature of less than $\sim 1500 \mathrm{~K}$ can reach $K=1$, and a quantitative visual divide based on reaction enthalpies and entropies distringuishes between reactions driven with electrochemistry versus those driven with thermochemistry. Note that the stoichiometry depicted in the legend is for readability; all reactions have been scaled to have the same number of electrons for best comparison (Supplemental Derivation S7). To be consistent, chemical formulas were used for all species in the legend, but the following formulas are not unique and refer to the specified chemical: $\mathrm{C}_{3} \mathrm{H}_{6} \mathrm{O}$ (propanal), $\mathrm{C}_{2} \mathrm{H}_{4} \mathrm{O}$ (ethylene oxide), $\mathrm{C}_{3} \mathrm{H}_{6}$ (propene), $\mathrm{C}_{6} \mathrm{H}_{6}$ (benzene), $\mathrm{C}_{3} \mathrm{H}_{3} \mathrm{~N}$ (acrylonitrile), and $\mathrm{C}_{6} \mathrm{H}_{8} \mathrm{~N}_{2}$ (adiponitrile). Raw thermodynamic data given in Supplemental Data S1 and S2 32, 33, 34, 35.

specific properties such as kinetics and selectivity must be taken into account when choosing a driving force, as well. Note that throughout our analysis we restrict ourselves to redox reactions for synthe- 515 sizing chemicals and do not address reactions where energy is extracted (e.g., via a combustion engine or a fuel cell).

On these proposed axes (Figure 5), the thermodynamic equilibrium conversion at ambient condi- 520 tions is readily determined for any reaction by generating a single point $(x, y)$. Unlike binary descriptors for a chemical reaction such as endo- vs. exothermic or sign of $\Delta G_{\mathrm{rxn}}$ (Supplemental Derivation S8), the points representing each reaction can ${ }_{525}$ be easily shifted to account for non-ambient conditions (e.g., operating conditions). Additionally, these points inherently encompass multiple discriminating properties of a chemical reaction. First, we can use these points to determine if temperature or 530 pressure can individually drive a reaction to high conversion via the $x$ and $y$ values (see Supplemental Derivation S6 for more details). Second, we can determine if, at practical operating conditions (e.g., elevated temperatures), other driving forces such as 535 pressure can result in high equilibrium conversion. Last, we can visually discriminate when voltage is necessary for high conversion, recognizing that for cases where temperature, pressure, or voltage all exhibit high fidelity as driving forces for a chemical reaction, significant practical maturity in the use of temperature and pressure as driving forces for a wide range of chemical reactions may favor their usage.

In addition to allowing direct comparison of chemical reactions, these axes also enable facile addition and subtraction of reactions. For example, both water splitting (blue diamond) and ammonia synthesis (pink diamond) are represented, but the sum of these two reactions, converting water and nitrogen to ammonia and oxygen, is also shown (blue circle) and has $(x, y)$ coordinates that are simply the sum of the two individual reaction points (Figure 6). This is a manifestation of Hess's Law, namely that the total enthalpy change for a reaction given by multiple steps is the sum of all enthalpy changes of the individual steps. Combining reactions on these axes to generate new reactions is therefore simple and enables quick visual analysis of how multiple reactions can work together from a thermodynamic perspective. This additive property of these axes makes it clear why using water as a source of hydrogen or oxygen can be difficult 
with pressure and temperature but is feasible with voltage as a driving force; since the water splitting point is so far from the vertical axis (very endothermic), the specific reaction where we want to replace hydrogen or oxygen would need to be equally far on the opposite side of $x=0$ to be thermochemically feasible using water as a reactant.

\section{Conclusions}

Beginning with the question: "why should a given chemical reaction be driven preferentially with temperature (thermal energy), pressure (mechanical energy), or voltage (electrical energy)?" we developed a non-dimensional, reactionindependent expression for chemical equilibrium as a function of thermodynamic driving forces. We then analyzed the thermodynamics for multiple industrial and lab-scale chemical reactions that rely on different combinations of temperature, pressure, and voltage as driving forces and compared them visually on the same axes, finding a clear discrimination between electrochemically and thermochemically driven reactions. Converting from temperature, pressure, and voltage to heat and work fluxes reveals that our analysis has a strong physical basis in work and energy exchanges.

The universal equation and facile visualization of chemical reactions provide both a quantitative jus-

565 tification for thermodynamic driving force as well as an intuitive platform for comparing multiple reactions. However, chemical reaction conditions are often dictated by more than just thermodynamics and require knowledge of kinetics, selectivity, costs,

570 and associated unit operations, such as those in- 615 volved in separations. Other parameters such as reactant availability, the possibility of modular and distributed manufacturing, and safety are also necessary considerations. Thus, the decision between 575 using traditional heat and mechanical work to drive a reaction versus using electricity in reality depends on much more than the thermodynamics. However, 620 academic and industrial research on chemical reactions often begins long before estimates of practical operating parameters are available. Quantitative thermodynamic visualizations of the type presented here can allow for comparing candidate reactions and driving forces at the early stages of developing 625 new reactions and processes, before a process is sufficiently mature to inform detailed technoeconomic and safety analyses.

\section{Methods}

Thermodynamic data was taken from NIST [32, the Dortmund Data Bank 33], Lange's Handbook of Chemistry 34, and group additivity theory via RMG 35 depending on availability, with the most recent data point used if multiple data points were provided (raw data provided in Supplemental Table S1). Derivations for all analytical thermo595 dynamic expressions shown in the main text are provided in the Supplemental Derivations S6 and S7. Starting with the expression for an equilibrium constant (equation 3), we derived an expression that explicitly included the dependence on specific driving forces of interest (pressure, temperature, and voltage). This expression was then nondimensionalized in preparation for better comparison and visualization. Energy and work exchanges were calculated by conducting energy and entropy balances. Analysis and visualization of all data was performed using Python and matplotlib. Prior to publication, this code will be hosted on Zenodo and be identifiable with a DOI URL.

\section{Supplemental Information}

Document S1: Supplemental derivations and supplemental tables S1-S2.

\section{Acknowledgments}

This material is based on work supported by the National Science Foundation under grant no. 1944007. The authors thank Nathan Corbin, Nikifar Lazouski, and Kindle Williams for useful discussions. ZJS and AML also acknowledge a graduate research fellowship from the National Science Foundation under Grant No. 1745302.

\section{Author Contributions}

Conceptualization, Z.J.S., and K.M.; Methodology, Z.J.S., A.M.L., and K.M.; Software, Z.J.S.; Validation, Z.J.S., A.M.L., and K.M.L.; Investigation, Z.J.S., A.M.L, and K.M.; Resources, Z.J.S., A.M.L., and K.M.; Writing - Original Draft, Z.J.S.; Writing-Review \&Editing, Z.J.S., A.M.L, and K.M.; Visualization, Z.J.S.; Supervision, K.M.; Funding Acquisition, K.M. 


\section{Declaration of Interests}

The authors declare no competing interests.

\section{References}

[1] EIA, International Energy Outlook 2016, Tech. rep. (2016).

[2] IEA, ICCA, DECHEMA, Technology Roadmap: Energy and GHG Reductions in the Chemical Industry ${ }^{700}$ via Catalytic Processes, Tech. rep. (2013).

[3] C. M. Friend, B. Xu, Heterogeneous Catalysis: A Central Science for a Sustainable Future Accounts of Chemical Research 50 (3) (2017) 517-521. doi:10.1021/acs.accounts.6b00510 URL http://pubs.acs.org/doi/abs/10.1021/acs. accounts.6b00510

[4] A. Arora, A. Gambardella, Implications for Energy Innovation from the Chemical Industry in: Accelerating Energy Innovation: Insights from Multiple Sectors, University of Chicago Press, 2011, pp. 87-111. doi:10.7208/chicago/9780226326856.001.0001 URL http://www.nber.org/chapters/c11751

[5] K. M. van Geem, V. V. Galvita, G. B. Marin, Making chemicals with electricity, Science 364 (6442) (2019) 734-735. doi:10.1126/science.aax5179.

[6] S. Gu, B. Xu, Y. Yan, Electrochemical Energy Engineering: A New Frontier of Chemical Engineering Innovation, Annual Review of Chemical and Biomolecular Engineering 5 (1) (2014) 429-454. doi:10.1146/ annurev-chembioeng-060713-040114

[7] C. Schnuelle, J. Thoeming, T. Wassermann, P. Thier A. v. Gleich, S. Goessling-Reisemann, Socio-technicaleconomic assessment of power-to-X: Potentials and limitations for an integration into the German energy system, Energy Research \& Social Science 51 (2019) 187197.

[8] Z. J. Schiffer, K. Manthiram, Electrification and Decarbonization of the Chemical Industry Joule 1 (1) (2017) 10-14. doi:10.1016/j.joule.2017.07.008 URL http://dx.doi.org/10.1016/j.joule.2017.07. 008

[9] G. G. Botte, Electrochemical Manufacturing in the Chemical Industry Interface magazine 23 (3) (2014) 49-55. doi:10.1149/2.F04143if URL http://interface.ecsdl.org/cgi/doi/10.1149/ 2.F04143if

[10] Z. Wang, R. R. Roberts, G. F. Naterer, K. S. Gabriel, Comparison of thermochemical, electrolytic, photoelec-

675 trolytic and photochemical solar-to-hydrogen production technologies International Journal of Hydrogen Energy 37 (21) (2012) 16287-16301. doi:10.1016/j. ijhydene.2012.03.057. 口 URL http://dx.doi.org/10.1016/j.ijhydene.2012.

680

[11] M. Jouny, W. Luc, F. Jiao, General Techno-Economic Analysis of $\mathrm{CO} 2$ Electrolysis Systems, Industrial and Engineering Chemistry Research 57 (6) (2018) 21652177. doi:10.1021/acs.iecr.7b03514

685 [12] Y. Zhao, B. P. Setzler, J. Wang, J. Nash, T. Wang, ${ }^{750}$ B. Xu, Y. Yan, An Efficient Direct Ammonia Fuel Cell for Affordable Carbon-Neutral Transportation, Joule (2019) 1-13doi:10.1016/j.joule.2019.07.005
URL https://linkinghub.elsevier.com/retrieve/ pii/S2542435119303216

[13] J. Na, B. Seo, J. Kim, C. W. Lee, H. Lee, Y. J. Hwang, B. K. Min, D. K. Lee, H. S. Oh, U. Lee, General technoeconomic analysis for electrochemical coproduction coupling carbon dioxide reduction with organic oxidation Nature Communications 10 (1) (2019). doi:10.1038/s41467-019-12744-y

URL http://dx.doi.org/10.1038/ s41467-019-12744-y

[14] K. Muller, K. Brooks, T. Autrey, Hydrogen Storage in Formic Acid: A Comparison of Process Options, Energy Fuels 31 (2017) 12603-12611. doi:10.1021/acs. energyfuels.7b02997

[15] M. Jouny, G. S. Hutchings, F. Jiao, Carbon monoxide electroreduction as an emerging platform for carbon utilization Nature Catalysis 2 (12) (2019) 1062-1070. doi: $10.1038 / s 41929-019-0388-2$

http://dx.doi.org/10.1038/ s41929-019-0388-2

[16] S. Verma, S. Lu, P. J. Kenis, Co-electrolysis of CO2 and glycerol as a pathway to carbon chemicals with improved technoeconomics due to low electricity consumption Nature Energy 4 (6) (2019) 466-474. doi:10.1038/s41560-019-0374-6

URL http://dx.doi.org/10.1038/ s41560-019-0374-6

[17] S. Carnot, Réflexions sur la puissance motrice du feu et sur les machines propres à développer cette puissance, Paris, 1824.

[18] J. Smeaton, XVIII. An experimental enquiry concerning the natural powers of water and wind to turn mils, and other machines, depending on a circular motion., Philosophical Transactions of the Royal Society 51 (1759) 100-174.

[19] K. H. Hoffmann, An Introduction to Endoreversible Thermodynamics (2008) 1-18doi:10.1478/ C1S0801011.

[20] B. Andresen, R. S. Berry, A. Nitzan, P. Salamon, Thermodynamics in finite time. I. The step-Carnot cycle, Physical Review A 15 (5) (1977) 2086-2093. doi: 10.1103/PhysRevA.15.2086

[21] S. Sieniutycz, J. Jeżowski, Energy limits for thermal engines and heat pumps at steady states, 2018. doi: 10.1016/b978-0-08-102557-4.00003-7

[22] G. Inzelt, Crossing the bridge between thermodynamics and electrochemistry. From the potential of the cell reaction to the electrode potential, ChemTexts 1 (1) (2015) 1-11. doi:10.1007/s40828-014-0002-9

[23] E. Keszei, Chemical Thermodynamics: An introduction. doi:10.1017/CB09781107415324.004

[24] E. Clarke, D. Glew, Evaluation of Thermodynamic Functions from Equilibrium Constants, Transactions of the Faraday Society 62 (1966) 539-547.

[25] J. O. Bockris, Comprehensive Treatise of Electrochemistry Vol. 3: Electrochemical Energy Conversion and Storage, 1981. doi:10.1007/978-1-4615-6687-8\{\_\}8

[26] T. Hecksher, Insights through dimensions, Nature Physics 13 (10) (2017) 1026. doi:10.1038/nphys4285

[27] J. Meixner, Coldness and temperature, Archive for Rational Mechanics and Analysis 57 (3) (1975) 281-290. doi:10.1007/BF00280159

[28] N. M. Bazhin, Mechanism of electric energy production in galvanic and concentration cells, Journal of Engineering Thermophysics 20 (3) (2011) 302-307. doi: 
10.1134/S1810232811030076

755 [29] N. M. Bazhin, V. N. Parmon, Conversion of the chemical reaction energy into useful work in the Van't Hoff equilibrium box, Journal of Chemical Education 84 (6) (2007) 1053-1055. doi:10.1021/ed084p1053

[30] A. Kowalewicz, Fuel-cell: power for future, Journal of KONES 8 (3) (2001) 325-333.

[31] A. Z. Panagiotopoulos, Essential Thermodynamics, 1st Edition, CreateSpace Independent Publishing Platform, 2011.

32] D. Burgess, Thermochemical Data, in: P. Linstrom, W. Mallard (Eds.), NIST Chemistry WebBook, NIST Standard Reference Database Number 69, National Institute of Standards and Technology, Gaithersburg, MD, Ch. Thermochem. doi:10.18434/T4D303

[33] Dortmund Data Bank (2020). URL www.ddbst . com

[34] N. A. Lange, Lange's Handbook of Chemistry, 15th Edition, McGraw-Hill, Inc., 1999.

[35] C. W. Gao, J. W. Allen, W. H. Green, R. H. West,

- Reaction Mechanism Generator: Automatic construc-

775 tion of chemical kinetic mechanisms Computer Physics

n Communications 203 (2016) 212-225. doi:10.1016/j. cpc.2016.02.013

URL http://dx.doi.org/10.1016/j.cpc.2016.02.013 